\title{
Rapid Regression of Exudative Maculopathy in Idiopathic Retinitis, Vasculitis, Aneurysms and Neuroretinitis Syndrome after Intravitreal Ranibizumab
}

\author{
Cristina Marín-Lambíes ${ }^{a}$ Roberto Gallego-Pinazo ${ }^{a}$ \\ David Salom $^{a}$ Javier Navarrete ${ }^{c}$ Manuel Díaz-Llopis ${ }^{a, b}$ \\ ${ }^{a}$ Department of Ophthalmology, University and Polytechnic Hospital La Fe, and \\ ${ }^{b}$ Faculty of Medicine, Valencia University, Valencia, and ${ }^{\mathrm{C}}$ Department of \\ Ophthalmology, La Ribera University Hospital, Alzira, Spain
}

\section{Key Words}

Idiopathic retinitis, vasculitis, aneurysms and neuroretinitis syndrome $\cdot$ Exudative maculopathy $\cdot$ Ranibizumab

\begin{abstract}
The idiopathic retinitis, vasculitis, aneurysms and neuroretinitis syndrome is a rare retinal vascular disorder characterized by multiple leaking aneurysmal dilations, retinal vasculitis, neuroretinitis and peripheral vascular ischemia. Visual loss mainly occurs due to the development of retinal neovascularization and/or exudative maculopathy. Although the treatment of choice has not yet been established, retinal photocoagulation seems to be the best option to control the disease and to prevent its progression. Herein, we report a case of idiopathic retinitis, vasculitis, aneurysms and neuroretinitis syndrome with both retinal neovascularization and macular exudation successfully managed with intravitreal ranibizumab (Lucentis ${ }^{\circledR}$ ) as adjunctive therapy to retinal photocoagulation.
\end{abstract}

\section{Introduction}

The idiopathic retinitis, vasculitis, aneurysms and neuroretinitis (IRVAN) syndrome is a retinal vascular disorder of unknown etiology characterized by three major criteria (multiple leaking aneurysmal dilations, retinal vasculitis and neuroretinitis) and three minor criteria (peripheral capillary nonperfusion, retinal neovascularization and macular exudation) [1]. IRVAN syndrome may progress rapidly to severe visual loss, 
mainly due to the development of exudative maculopathy and/or retinal ischemia, and its neovascular sequelae such as vitreous hemorrhage and neovascular glaucoma $[1,2]$.

Retinal photocoagulation (RP) is the only treatment that has shown evidence to prevent the progression of the disease by acting on proliferative changes $[1,3]$. Herein, we report a clinical case of IRVAN syndrome with both retinal neovascularization and macular exudation successfully managed with intravitreal ranibizumab (Lucentis ${ }^{\circledR}$ ) as adjunctive therapy to RP.

\section{Materials and Methods}

A 42-year-old man came to our department with a 1-month history of blurred vision in his right eye (RE). There was no previous history of systemic illness or ocular problems. Best corrected visual acuity (BCVA) was 20/40 in the RE and 20/20 in left eye (LE). Slit lamp examination was bilaterally unremarkable. Fundus examination of the RE evidenced the presence of numerous lipid and soft exudates within the macular area, accompanied by tortuous vessels with aneurysmal changes and marked papillary neovascularization (fig. 1a). LE funduscopy revealed asymptomatic papillary neovascularization, lipid and soft exudates in the vascular arcades, but no macular involvement (fig. 1b). Fluorescein angiography demonstrated bilateral aneurysmal changes of the retinal arteries with staining of their walls, papillary neovascularization with active leakage of the optical nerve head, and extensive areas of retinal ischemia not only in the periphery but also in the posterior pole (fig. 1c, d). Optical coherence tomography (OCT; Carl Zeiss, Dublin, Calif., USA) showed a subfoveal detachment of the neurosensory retina with prominent reflectance from the accumulation of lipid exudates in the outer retina of the RE and a normal foveal contour in the LE (fig. 1e, f).

A complete ocular and systemic examination including chest X-ray, routine blood chemistry, erythrocyte sedimentation rate, angiotensin-converting enzyme, purified protein derivative, venereal disease research laboratory, fluorescent treponemal antibody absorption test, antiphospholipid antibodies, antinuclear antibodies and antineutrophil cytoplasmic antibodies was performed, and the Mantoux and pathergy tests were within normal limits. Given the clinical findings, a diagnosis of IRVAN syndrome was made.

\section{Results}

Two monthly intravitreal injections of $0.5 \mathrm{mg}$ ranibizumab (Lucentis) were bilaterally administered. Optic nerve neovascularization and macular exudation dramatically regressed 1 month after the second injection, and BCVA improved to 20/30 in the RE (fig. 2a, b). Panretinal photocoagulation (PRP) in the RE and selective photocoagulation of the ischemic areas in the LE were performed (fig. 2c-f). At the 1year follow-up, BCVA was 20/20 bilaterally, with complete regression of neovascularization and exudation and no need for further retreatment.

\section{Discussion}

IRVAN syndrome is a retinal vascular disease characterized by bilateral multiple leaking aneurysmal dilations, vasculitis, neuroretinitis and peripheral vascular ischemia. Very few cases have been reported in the literature since Kincaid and Schatz [4] described the first 2 patients with bilateral retinal arteritis and multiple aneurysmal dilatations in 1983. More than 10 years later, Chang et al. [1] were the first to 
characterize this syndrome as IRVAN. So far, publications about this syndrome are isolated cases or small case series.

The disease usually presents in the third or fourth decade of life and there are no known systemic associations. Visual loss is caused by exudative maculopathy and/or neovascular sequelae of retinal ischemia so that the prognosis of IRVAN syndrome depends on prompt treatment $[1,2]$. Although the best therapy has not yet been established, RP seems to be the most suitable option to control the disease and to prevent its progression [1-3]. Other treatments have been reported with variable results such as corticosteroids [1-3], pars plana vitrectomy [2, 5], anti-TNF alpha agents [6], antiangiogenic drugs [7, 8] or the direct application of laser on aneurysms [9].

A functional staging system based on progressive ischemia and its sequelae has been proposed in order to improve the treatment algorithm [2]. Stage 1 includes macroaneurysms, exudation, neuroretinitis and retinal vasculitis; stage 2 includes angiographic evidence of capillary nonperfusion; stage 3 is characterized by the presence of posterior segment neovascularization and/or vitreous hemorrhage; stage 4 includes anterior segment neovascularization, and stage 5 includes neovascular glaucoma.

Samuel et al. [2] published the most relevant case series of IRVAN syndrome, including 44 eyes of 22 patients. All eyes treated with laser in stage 2 maintained a vision of 20/20 without progression of the disease. Many of the treated eyes in stage 3 also maintained an acceptable visual acuity of $20 / 40$ or better; however, $25 \%$ of the patients in this stage ended with a visual acuity of $20 / 200$ or worse, with progression of the disease. Half of the patients treated in stages 4 and 5 progressed to a severe vision loss in spite of treatment. These results show the importance of an early diagnosis and treatment in patients with IRVAN syndrome; the treatment of stage 2 cases being the one that achieves better visual results.

Vascular endothelial growth factor plays an important role in ocular neovascularization as ischemia is the main stimulus in its production. Antiangiogenic drugs have demonstrated a beneficial effect by controlling neovascularization and macular edema in diseases such as exudative age-related macular degeneration [10], diabetic retinopathy [11] and retinal vein occlusion [12]. The use of these drugs has also been described in retinal vascular diseases that share certain characteristics with IRVAN syndrome, such as retinal arterial macroaneurysms [13], Eales' disease [14] and Coats' disease [15]. Previous clinical case reports have shown the efficacy of intravitreal bevacizumab and ranibizumab therapy as adjunctive treatment for IRVAN syndrome; however, none of these cases had exudative maculopathy $[7,8]$.

We reported a clinical case of a stage 3 IRVAN syndrome with the presence of macroaneurysms, exudation, neuroretinitis, retinal vasculitis and bilateral retinal neovascularization. Two monthly intravitreal injections of ranibizumab (Lucentis) were administered in each eye. One month after the treatment, rapid regression of exudative maculopathy was evidenced. PRP in the RE and selective laser application on the ischemic areas in the LE were consecutively performed. After the 1-year follow-up, visual acuity remained $20 / 20$ in both eyes, with no evidence of progression of the disease and no need for further retreatment. 
The case presented underlines that intravitreal ranibizumab alone can cause a reduction of the IRVAN-associated exudation. We conclude that ranibizumab constitutes an effective and safe adjunctive treatment for IRVAN syndrome, contributing to the control and disappearance not only of the neovascularization but also of the macular exudates. Further studies are needed to confirm our observations.

\section{Funding}

The authors have not received financial support for this paper.

\section{Disclosure Statement}

The authors declare that there are no conflicts of interest relevant to this paper. 

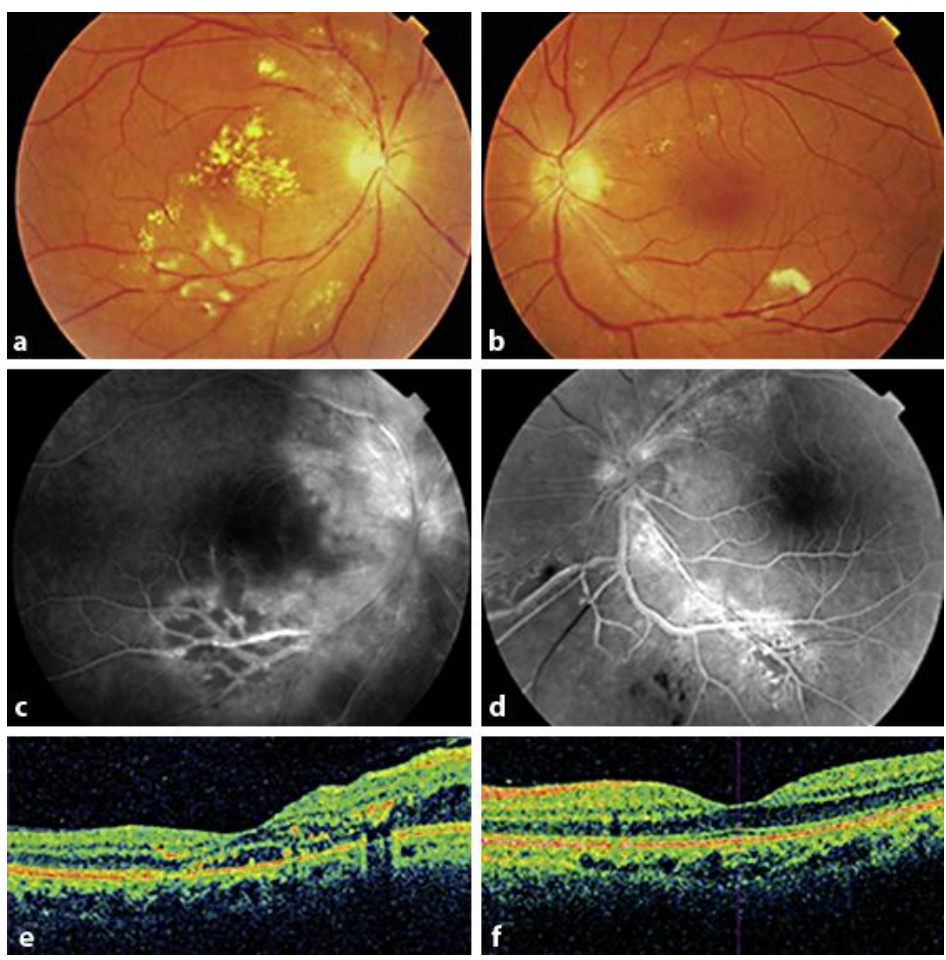

Fig. 1. Fundus examination of the RE evidences the presence of numerous lipid and soft exudates involving the macular area, aneurysmal changes and marked peripapillary neovascularization (a). LE funduscopy reveals asymptomatic papillary neovascularization, lipid exudates in the superior arcade and soft exudates in the inferior arcade, but no macular involvement (b). Fluorescein angiography demonstrates bilateral aneurysmal changes of the retinal arteries with staining of their walls, papillary neovascularization with active leakage of the optical nerve head, and extensive areas of retinal ischemia not only in the periphery but also in the posterior pole $(\mathbf{c}, \mathbf{d})$. OCT showed a subfoveal detachment of the neurosensory retina with prominent reflectance from the accumulation of lipid exudates in the outer retina of the RE (e) and no macular exudation in the LE (f). 

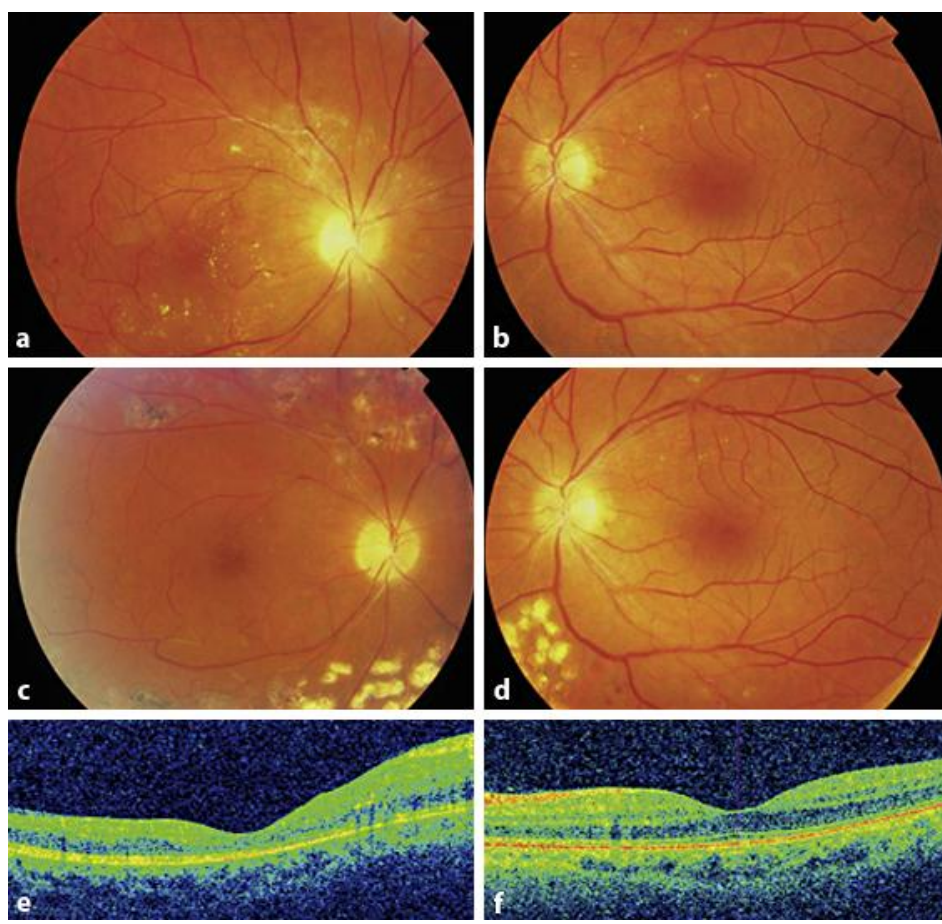

Fig. 2. After 2 monthly intravitreal injections of $0.5 \mathrm{mg}$ ranibizumab, regression of the heavy lipid exudation was observed in both eyes $(\mathbf{a}, \mathbf{b})$. PRP in the RE and selective RP of the ischemic areas in the LE were performed, achieving complete regression of exudation and neovascularization (c, d). After treatment, OCT showed a normal foveal contour in both eyes with regression of the lipid exudates in the RE (e, f).

\section{References}

$\checkmark 1$ Chang TS, Aylward GW, Davis JL, Mieler WF, Oliver GL, Maberley AL, Gass JD: Idiopathic retinal vasculitis, aneurysms, and neuro-retinitis. Retinal Vasculitis Study. Ophthalmology 1995;102:10891097.

-2 Samuel MA, Equi RA, Chang TS, Mieler W, Jampol LM, Hay D, Yannuzzi LA: Idiopathic retinitis, vasculitis, aneurysms, and neuroretinitis (IRVAN): new observations and a proposed staging system. Ophthalmology 2007;114:1526-1529.

-3 Abu El-Asrar AM, Jestaneiah S, Al-Serhani AM: Regression of aneurysmal dilatations in a case of idiopathic retinal vasculitis, aneurysms and neuroretinitis (IRVAN) associated with allergic fungal sinusitis. Eye (Lond) 2004;18:197-199.

4 Kincaid J, Schatz H: Bilateral retinal arteritis with multiple aneurysmal dilatations. Retina 1983;3:171178.

5 Tomita M, Matsubara T, Yamada H, Takahashi K, Nishimura T, Sho K, Uyama M, Matsumara M: Long term follow up in a case of successfully treated idiopathic retinal vasculitis, aneurysms, and neuroretinitis (IRVAN). Br J Ophthalmol 2004;88:302-303.

6 6 Cheema RA, Al-Askar E, Cheema HR: Infliximab therapy for idiopathic retinal vasculitis, aneurysm, and neuroretinitis syndrome. J Ocul Pharmacol Ther 2011;27:407-410.

-7 Karagiannis D, Soumplis V, Georgalas I, Kandarakis A: Ranibizumab for idiopathic retinal vasculitis, aneurysms, and neuroretinitis: favorable results. Eur J Ophthalmol 2010;20:792-794.

-8 Akesbi J, Brousseaud FX, Adam R, Rodallec T, Nordmann JP: Intravitreal bevacizumab (Avastin) in idiopathic retinitis, vasculitis, aneurysms and neuroretinitis. Acta Ophthalmol 2010;88:40-41.

9 Gedik S, Yilmaz G, Akça S, Akova YA: An atypical case of idiopathic retinal vasculitis, aneurysms, and neuroretinitis (IRVAN) syndrome. Eye (Lond) 2005;19:469-471. 
10 Boyer DS, Antoszyk AN, Awh CC, Bhisitkul RB, Shapiro H, Acharya NR, MARINA Study Group: Subgroup analysis of the MARINA study of ranibizumab in neovascular age-related macular degeneration. Ophthalmology 2007;114:246-252.

11 Mitchell P, Bandello F, Schmidt-Erfurth U, Lang GE, Massin P, Schlingemann R0, Sutter F, Simader C, Burian G, Gerstner O, Weichselberger A, RESTORE study group: The RESTORE study: ranibizumab monotherapy or combined with laser versus laser monotherapy for diabetic macular edema. Ophthalmology 2011;118:615-625.

-12 Brown DM, Campochiaro PA, Singh RP, Li Z, Gray S, Saroj N, Rundle AC, Rubio RG, Murahashi WY, Cruise Investigators: Ranibizumab for macular edema following central retinal vein occlusion: 6-month primary end point results of a phase III study. Ophthalmology 2010;117:1124-1133.

13 Wenkstern AR, Petersen H: Intravitreal ranibizumab in retinal macroaneurysm. Graefes Arch Clin Exp Ophthalmol 2010;248:1667-1670.

14 Kumar A, Sinha S: Rapid regression of disc and retinal neovascularization in a case of Eales disease after intravitreal bevacizumab. Can J Ophthalmol 2007;42:335-336.

15 Diago T, Valls B, Pulido JS: Coats' disease associated with muscular dystrophy treated with ranibizumab. Eye (Lond) 2010;24:1295-1296. 